\title{
Experimental Results for Over-the-Horizon Planetary Exploration using a LIDAR sensor
}

\author{
Ioannis Rekleitis ${ }^{1}$, Jean-Luc Bedwani ${ }^{2}$, David Gingras ${ }^{2}$, and Erick Dupuis ${ }^{2}$ \\ 1 School of Computer Science, McGill University, Montreal, Canada. yiannis@cim.mcgill.ca \\ 2 Space Technologies, Canadian Space Agency, St. Hubert, Canada. FirstName.LastName@space.gc.ca
}

Summary. In this paper we present the experimental results validating the approach for autonomous planetary exploration developed by the Canadian Space Agency (CSA). The goal of this work is to autonomously navigate to remote locations, well beyond the sensing horizon of the rover, with minimal interaction with a human operator. We employ LIDAR range sensors due to their accuracy, long range and robustness in the harsh lighting conditions of space. Irregular triangular meshes (ITM) are used for representing the environment providing an accurate yet compact spatial representation. In this paper after a brief overview of the proposed approach, we discuss the terrain modelling used. A variety of experiments performed in CSA's Mars emulation terrain that validate our approach are also presented.

\section{Introduction}

Planetary exploration is one of the biggest challenges in robotics. The successes of Spirit and Opportunity, the Mars Exploration Rovers (MER), demonstrated the capabilities of robotics in space exploration [18]. The MER's limited autonomy capabilities restrict the distance at which the rovers can travel in a single command cycle. The next rover missions to Mars are the "Mars Science Laboratory" (MSL) [27] and ESA's ExoMars [26]. Both of these missions have set target traverse distances on the order of one kilometer per day. Therefore, they are expected to regularly drive a significant distance beyond the horizon of their environment sensors. Earth-based operators will therefore not know a-priori the detailed geometry of the environment, and thus will not be able to select way-points for the rovers throughout their traverses. The problem of autonomous long range navigation is also very important in terrestrial settings. The DARPA grand challenge in 2005 resulted in several vehicles travelling more than $200 \mathrm{~km}$ over desert terrain [20]. Similar work involved traverses on the order to $30 \mathrm{~km}$ in the Atacama desert [7] using vision. See also [6] for a discussion of the many challenges and more related work.

At the Canadian Space Agency (CSA) we have developed an integrated approach to address the needs for Over-the-Horizon autonomous navigation; which is defined as a traverse that takes the rover beyond the horizon of the rover's environment sensors. The key components of the planetary exploration capabilities are: terrain sensing and environmental modelling, path-planning, and trajectory following.

In the next Section we present some related work. Section 3 provides a brief outline of our approach in order to situate the experimental results presented. Section 4 presents an overview of our experimental setup; while Section 5 describes our terrain modelling approach and presents quantitative results on the efficiency of the mesh decimation. Section 6 contains results from a representative experiment and the paper concludes with lessons learned and future work.

\section{Related Work}

The work on planetary exploration can be divided according to the sensing modality used and also according to the environment representation used. Both vision $[19,10,15]$ and LIDAR $[12,3]$ technologies have 


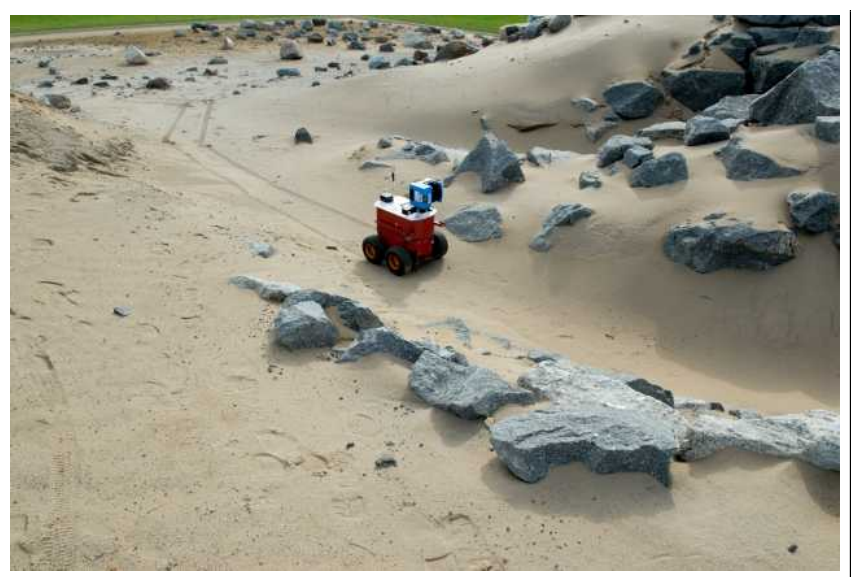

(a)

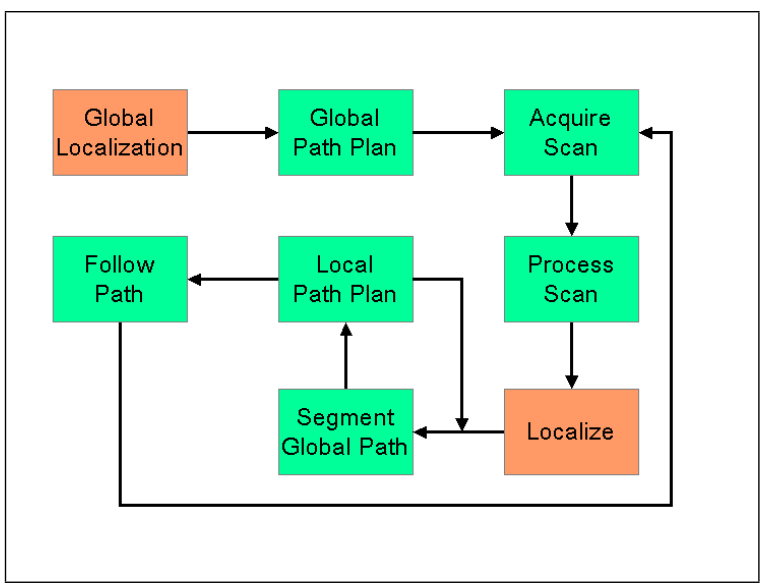

(b)

Fig. 1. (a) The Mars terrain with our modified P2-AT robot. (b) The main components of our proposed autonomous Over-the-Horizon navigation process.

been proposed, each one having different advantages and disadvantages. Early work on planetary exploration using LIDAR [12, 3], though promising, was not compatible with the weight constraints. The Mars Exploration Rovers are currently performing long traverses using stereo vision [11, 28]. Stereo cameras although lightweight, require more computing power, have limited range and accuracy. Currently, LIDAR based systems ${ }^{3}$ have been successfully used in space missions and thus are space qualified. The major advantage of LIDAR systems is their superior resolution and range.

Several types of exploration methods have been considered for autonomous planetary exploration. In the area of path-planning, a bug-like algorithm was proposed for micro-rovers [16], while potential-field like methods were also suggested [24]. The autonomy aspects of planetary exploration have been discussed ranging from trajectory planning in simulation [29] to varying behaviors based on the vision sensor data collected [14] and for ensuring visual coverage [9]. Finally the problem of human to rover interactions has also been studied $[2,17]$.

Currently, the most advanced exploration robots that have been deployed for planetary exploration are the Mars Exploration Rovers (MER) "Spirit" and "Opportunity". These rovers have successfully demonstrated, on Mars, concepts such as visual odometry and autonomous path selection from a terrain model acquired from sensor data [4]. The models obtained through stereo imagery are used for both automatic terrain assessment and visual odometry.

In the case of automatic terrain assessment, the cloud of 3D points is used to evaluate the traversability of the terrain immediately in front of the rover, defined as a regular grid of square patches. In the case of visual odometry, the model is used to identify and track features of the terrain to mitigate the effect of slip [13].

For our work, we have been using, a laser range sensor (LIDAR) as the main sensing modality. Several factors have motivated the choice of a LIDAR sensor: among others, our mobility platform, a modified Pioneer P2-AT, has very low ground clearance. A LIDAR sensor is capable of providing range data to build terrain models with 1-2 cm accuracy. Such an accuracy would be difficult to attain with most stereo vision systems over the full range of measurement. It is worth noting that such accuracy is also very important for the scientific return of the mission. An additional advantage of LIDAR sensors is that they return accurate geometric information in three dimensions in the form of a 3D point cloud without requiring additional

\footnotetext{
${ }^{3}$ http://www.neptec.com http://www.optech.ca/ http://sm.mdacorporation.com/
} 
processing. Finally, since they do not rely on ambient lighting, we do not have to address the problems arising from adverse lighting conditions.

We are taking advantage of this sensor characteristic to build a terrain model that, not only preserves the geometry of the terrain, but it is also readily usable for path planning and navigation. The majority of previous work uses some form of grid representations, often in the form of an elevation map. Our approach utilizes an Irregular Triangular Mesh (ITM) to represent the environment. By allowing for variable resolution we are capable to preserve the surface details in the topographically interesting regions (obstacles) while achieving a very sparse representation over the flat areas, allowing for very efficient path planning.

\section{Technical Approach}

We operate under the assumption that a global map is available from satellite imagery, previous missions, or from data collected during descent [21, 25]. Figure 1b shows a flowchart of the complete exploration process. At top level, the rover uses the global map to plan a path from its current position to an operatorspecified location; the rover collects the first local scan using its LIDAR sensor, then the global path is incrementally segmented using the locally collected scans; each time an optimal trajectory is planned through the representation of the local scan. Finally, the rover uses the local path to navigate to the next way-point. At the current state, the pose estimation from the Inertial Measurement Unit (IMU) and the odometer, combined with a trajectory length less than $10 \mathrm{~m}$ allows to safely navigate in open loop without relocalizing between successive scans. Combining the LIDAR data with the pose estimate in a SLAM framework is currently under development and beyond the scope of this paper.

Central to our approach is the representation used for modelling the surrounding terrain. The LIDAR sensor returns a set of points in 3D. We used a Delaunay triangulation [5] in polar coordinates which results into an Irregular Triangular Mesh (ITM) [8] representing the sensed surface. The mesh is further decimated by combining several coplanar triangles into a single triangle. The decimation process uses a maximum acceptable error $\epsilon$, to ensure that all eliminated points are within $\epsilon$ from the resulting triangle. The implementation of the terrain modelling and decimation using a triangular mesh is done using the Visualisation Toolkit [1] libraries from Kitware Inc.

The use of ITMs for terrain modelling leads to a very efficient path planning approach. The dual graph of the triangular mesh is used for path planning. The dual graph is constructed in such a way that every node corresponds to a triangle and the nodes of adjacent triangles are connected through their common edge. Consequently, path planning is implemented as a graph search, either Dijkstra's algorithm or $\mathrm{A}^{*}$, on the dual graph. Different cost functions have been investigated taking into account the Euclidean distance to the destination, the slope of the terrain and also the roughness. For a detailed description of CSA's path planning approach please refer to [23].

\section{Experimental Setup}

Two different LIDAR sensors have been used for our experiments. For the 2006 testing season we used an ILRIS 3D unit from OPTECH ${ }^{4}$ with a range of over $1 \mathrm{~km}$ but with a field of view (fov) limited to $40^{\circ}$; cf. Fig. 2a. During the 2007 season, a new sensor developed in-house, was used. It consists of a SICK Laser Range Finder (LRF) mounted on a pan unit. The LRF scans vertical slices and by panning $360^{\circ}$ the sensor returns a complete scan of its surroundings. The range of the new sensor is maximum $30 \mathrm{~m}$. Figure $2 \mathrm{~b}$ shows the new sensor on our mobile platform during an experiment.

CSA's experimental terrain is a $60 \mathrm{~m}$ by $30 \mathrm{~m}$ testing area that is designed to emulate a broad variety of Martian topographies. Figures 1a and 2 show the robot in different locations on the terrain, while Fig. 5 presents a complete model of the terrain. The terrain is covered with sand and only intends to emulate the topography of some areas on Mars. The geotechnical properties of the soil are not meant to be representative of those on Mars.

\footnotetext{
${ }^{4}$ http://www.optech.ca/
} 


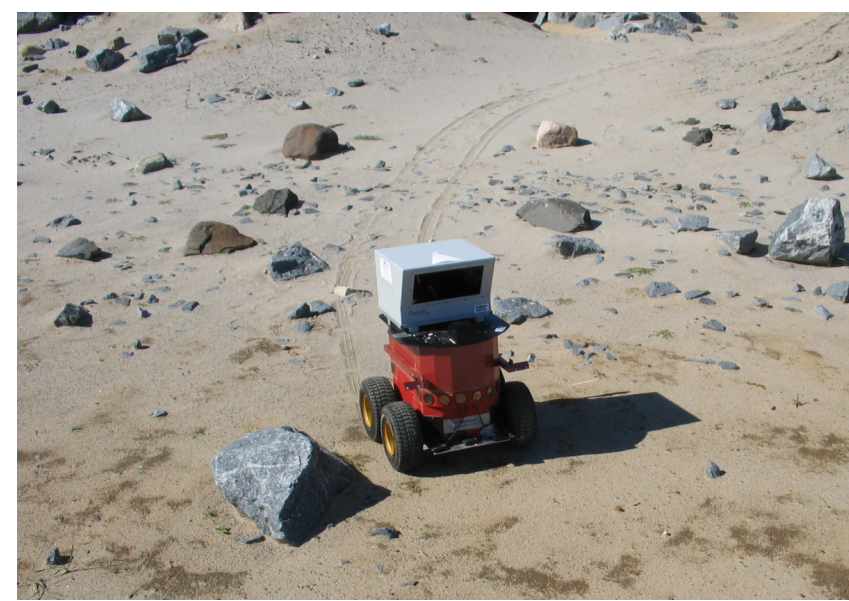

(a)

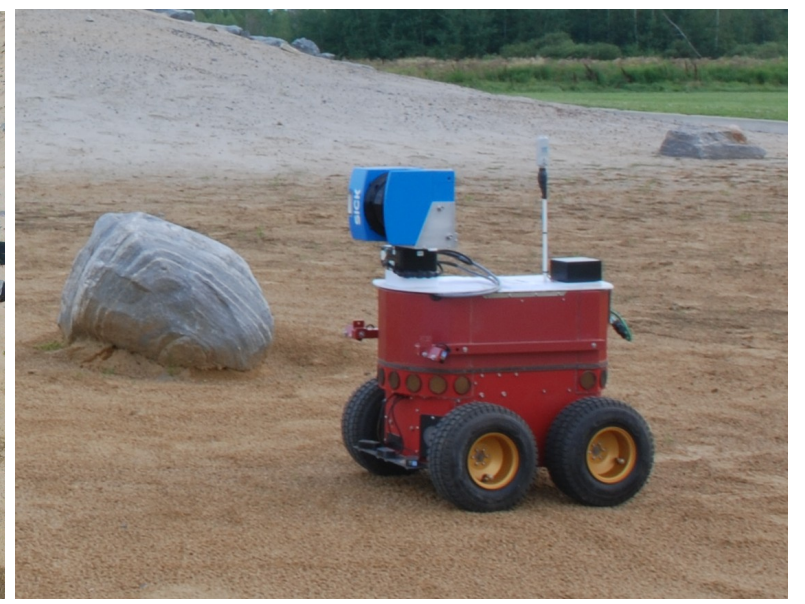

(b)

Fig. 2. (a) The ILRIS 3D sensor on top of the modified P2-AT robot. (b) The SICK sensor on a pan-unit mounted on the same robot.

The mobile robot base that was used to conduct the experiments is a P2-AT mobile robot from ActiveMedia; the robot is shown in Fig. 1a and Fig. 2. The P2-AT is a skid-steered four-wheeled robot. The robot comes equipped with motor encoders for odometry. It is also equipped with a 6-axis Inertial Measurement Unit (IMU) from Crossbow. The IMU provides angular velocity readings through three solid-state gyroscopes, and linear acceleration readings through three accelerometers. The gyroscopes are used to correct the odometry readings, which are very sensitive to slip during rotations. The accelerometers are used to reset the roll and pitch components of the robot's attitude by measuring the components of the gravitational acceleration vector. The robot is also equipped with a digital compass (TCM2 from PNI Corporation). The compass is used to reset the yaw component of the robot's attitude. The TCM2 is only used at rest since the motors induce magnetic fields that corrupt the sensor's readings while running. On Mars the compass would be replaced with a different orientation sensor, such as a Sun-sensor. The data from the wheel odometry, the IMU and the digital compass are fused together to provide a $6 \mathrm{DOF}$ state estimate.

After two years of rigorous testing, the robot failed due to the harsh conditions on the Mars-like terrain. In particular, the constant strain from sand, especially during rotations, resulted in several faults. The most important was that the shear pins in all four wheel-shafts broke. Because the shafts were press-fitted, the robot continued to operated because of friction, except when the resistance from the ground was increased. Moreover, the transmission belts were abraded and as a result in one side they had started to come apart. After the end of the 2007 season different improvements were implemented including adding bigger shafts, increasing the gear ration to obtain $50 \%$ more torque, and replacing the transmition belts with a chain drive using a sprocket mechanism in the pulleys. The modified version of the P2AT was capable this spring (2008) to negotiate steeper inclines and bigger obstacles.

\section{Terrain Modelling}

The LIDAR sensor is mounted on the robot scanning at a grazing angle with respect to the ground. As a result the resolution of the data varies with the distance from the robot, and obstacles leave long shadows with no data; cf. Fig. 3a,d. As mentioned earlier, the Delaunay triangulation is used to construct the Irregular Triangular Mesh (ITM) using polar coordinates. Triangles that appears inside the shadow areas were eliminated; cf. Fig. 3b,e. Due to the dense nature of the scans, the constructed ITMs had on average 61,700 triangles for the tests in 2006 and 216,000 triangles for 2007. As many of these triangles were coplanar they did not contributed any information to the terrain models; cf. Fig. 3c,f. The ITM's were decimated, as described earlier, using the VTK libraries. 


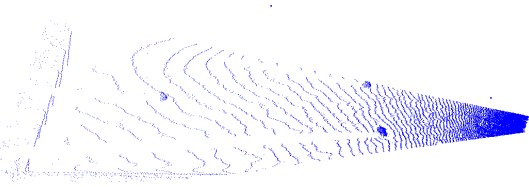

(a)

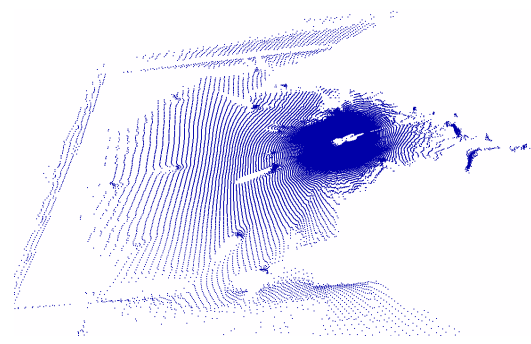

(d)

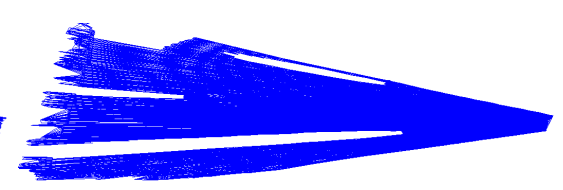

(b)

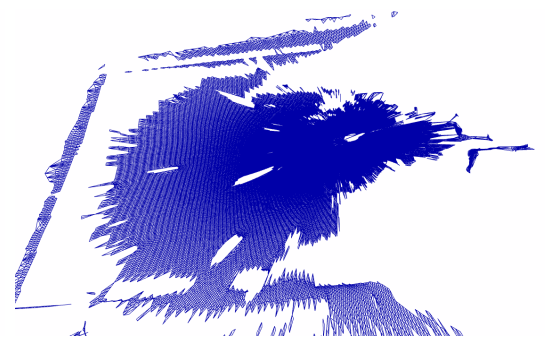

(e)

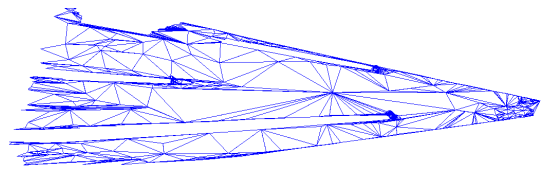

(c)

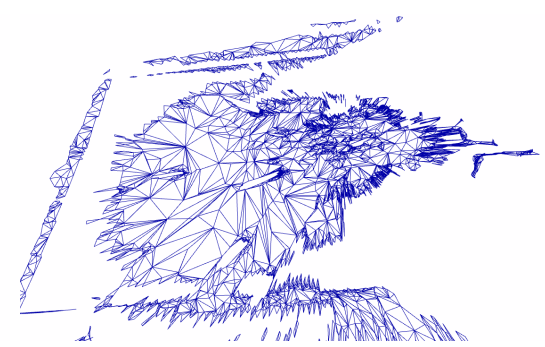

(f)

Fig. 3. (a) The raw data-points from the ILRIS 3D unit from OPTECH. (b) The ITM resulting from the Delaunay triangulation using the polar coordinates for proximity metric. (c) The decimated mesh for the same data. (d) The raw data-points from a $360^{\circ}$ scan of the SICK laser. (e) The ITM as in (b). (f) The decimated mesh of the data from (d).

During the testing season of 2006, 96 scans were collected from many different locations, cf. Fig. 4a. Due to the limited fov of the sensor, often, from the same position several scans in different orientations were collected. During the 2007 season 105 scans were collected, shown in Fig. 4b. Even though the two sets of scans were collected from different LIDAR sensors, the decimation ratios achieved were comparable. Table 1 presents the cumulative results for decimation ratios of $80 \%, 90 \%$, and $95 \%$. With acceptable error as low as $1.5 \mathrm{~cm}$, decimation ratios of up to $94.9 \%$ were achieved on average. It is worth noting that the 2007 scans had a $360^{\circ}$ fov, as such there were on average 111,000 data-points per scan compared to 31,200 points, on average per scan, for the $40^{\circ}$ fov scans collected with the ILRIS 3D LIDAR.

\begin{tabular}{|l|r|r|r|r|r|r|r|}
\hline & \multicolumn{5}{|c|}{ Target Decimation Ratio } \\
\hline & $\begin{array}{r}\text { Original } \\
\text { Scans }\end{array}$ & \multicolumn{2}{|c|}{$80 \%$} & \multicolumn{2}{c|}{$90 \%$} & \multicolumn{2}{c|}{$95 \%$} \\
\hline & & Number & Real \% & Number & Real \% & Number & Real \% \\
\hline 2006 Points (mean) & 31200 & 6530 & $79.00 \%$ & 3440 & $88.86 \%$ & 2090 & $93.09 \%$ \\
\hline 2006 Points (std) & 7840 & & $0.74 \%$ & & $1.16 \%$ & & $2.31 \%$ \\
\hline 2006 Triangles(mean) & 61700 & 12300 & $80.00 \%$ & 6190 & $89.91 \%$ & 3590 & $94.01 \%$ \\
\hline 2006 Triangles(std) & 15800 & & $0.00 \%$ & & $0.75 \%$ & & $1.90 \%$ \\
\hline 2007 Points (mean) & 111000 & 23400 & $78.91 \%$ & 12500 & $88.72 \%$ & 6700 & $93.69 \%$ \\
\hline 2007 Points (std) & 10300 & & $0.23 \%$ & & $0.27 \%$ & & $0.28 \%$ \\
\hline 2007 Triangles(mean) & 216000 & 43300 & $80.00 \%$ & 21600 & $90.00 \%$ & 10900 & $94.98 \%$ \\
\hline 2007 Triangles(std) & 20100 & & $0.00 \%$ & & $0.00 \%$ & & $0.14 \%$ \\
\hline
\end{tabular}

Table 1. Properties of Decimated Terrain Scans for the 2006 and 2007 testing seasons. Acceptable Error $1.5 \mathrm{~cm}$.

\section{Experimental Results}

The experiments were performed at CSA's Mars emulation terrain. During the 2006 testing campaign [22] several semi-autonomous navigation experiments were performed achieving trajectories up to $150 \mathrm{~m}$; cf. Fig. 


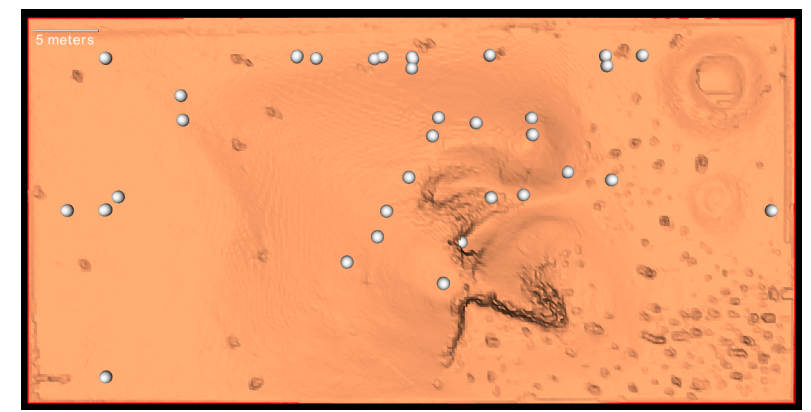

(a)

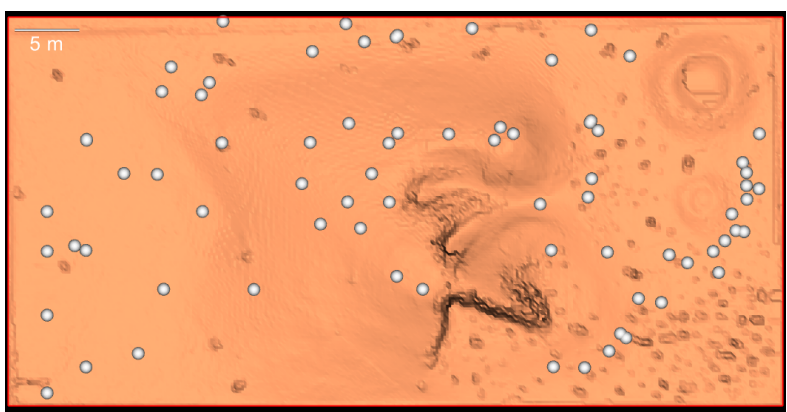

(b)

Fig. 4. (a) The locations from where 96 scans of $40^{\circ}$ fov were collected during the 2006 testing season. (b) The location of the 105 scans collected during the 2007 testing season.

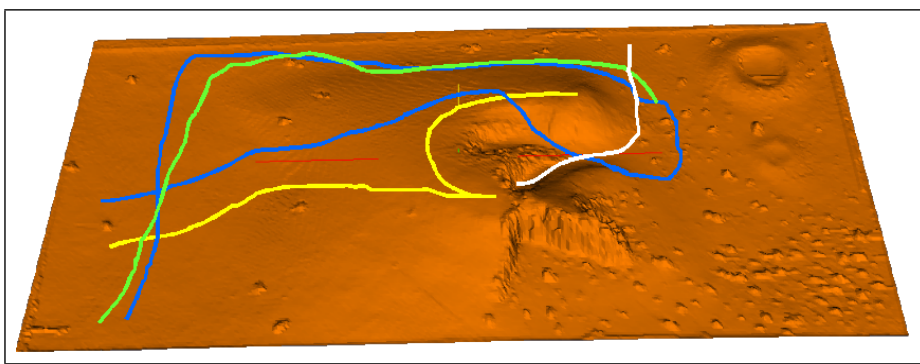

(a)

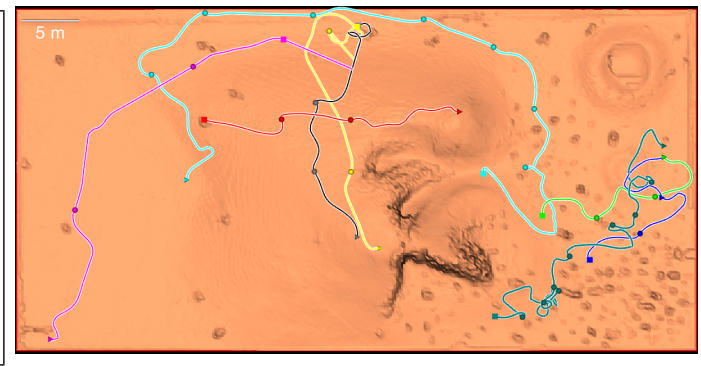

(b)

Fig. 5. (a) The 2006 testing season semi-autonomous Over-the-Horizon navigation trajectories on the Mars emulation terrain. (b) The 2007 testing season autonomous Over-the-Horizon navigation trajectories. The Mars emulation terrain depicted in the figures is $60 \mathrm{~m}$ by $30 \mathrm{~m}$.

5a. Last year (2007), fully autonomous experiments were conducted. The trajectories covered the majority of the terrain, as can be seen from figure $5 \mathrm{~b}$ where the trajectories from all the experiments are displayed.

The scans collected during the two testing seasons were also used to verify the quality of our pathplanning algorithm by off-line batch testing. For these experiments of path-planning on a single scan, the start position was always assumed to be the position from which the scan was acquired. The end-location was randomly selected to be at five or ten meters from the origin, and inside the Mars emulation terrain. If the destination point was unreachable, that is in an area of forbidding slope or with no data, then another point was selected randomly, up to three times. Out of 94 scans with recorded origin, acceptable destinations were found randomly for 82 scans at a distance of five meters, and 68 destinations at a distance of ten meters. Please note, that for every scan only 3 attempts were made to find a destination point randomly. The results are an indication of the challenging terrain where obstacles created shadows with no data and several areas had non-traversable slopes. Every time a destination point was inside the mesh and at a navigable slope, the path planner found a smooth path. Table 2 presents average results for the computed paths for destinations at five and ten meters, respectively. The computation time was on average 14 seconds for the destinations at five meters, and 25 seconds for ten meters. The proposed planning method was very efficient, the paths were computed in seconds using ITMs with several thousand triangles; cf. Table 2. Furthermore, the ITMs used were created from LIDAR scans of more than a hundred thousand points each; cf. Table 1 for the decimation rates. The computed path was on average $25 \%$ longer than a straight line between start and destination. It is worth noting that the estimated paths had to negotiate maximum slopes of $30^{\circ}$ on average, but the triangles with high slope were always very small. A weighted average slope $(W S)$ was calculated by multiplying the slope of each triangle on the path with the area that triangle and then normalized by dividing by the sum of all the areas: 


$$
W S=\frac{\sum S_{i} A_{i}}{\sum A_{i}}
$$

where $S_{i}$ and $A_{i}$ is the slope and the area of triangle $i$ respectively . As can be seen by the last column in Table 2, the average slope $(W S)$ is in the order of two degrees, as the path planner favored the more level ground.

\begin{tabular}{|l|c|c|c|c|c|c|c|}
\hline & $\begin{array}{c}\text { Time } \\
(\mathrm{sec})\end{array}$ & $\begin{array}{c}\text { Path } \\
\text { length }\end{array}$ & $\begin{array}{c}\text { Euclidean } \\
\text { distance }\end{array}$ & $\begin{array}{c}\text { \# of path } \\
\text { points }\end{array}$ & $\begin{array}{c}\text { \# of } \\
\text { triangles }\end{array}$ & $\begin{array}{c}\text { Max } \\
\text { slope }\end{array}$ & $\begin{array}{c}\text { Weighted } \\
\text { slope }(W S)\end{array}$ \\
\hline $5 \mathrm{~m}$ (mean) & 13.8 & $6.33 \mathrm{~m}$ & $5.0 \mathrm{~m}$ & 10 & 7744 & $27.2^{\circ}$ & $1.8^{\circ}$ \\
\hline $5 \mathrm{~m}$ (std) & 17.8 & $1.06 \mathrm{~m}$ & $0.13 \mathrm{~m}$ & 5.5 & 2330 & $22.8^{\circ}$ & $2.0^{\circ}$ \\
\hline $10 \mathrm{~m}$ (mean) & 25.4 & $12.70 \mathrm{~m}$ & $10.0 \mathrm{~m}$ & 14.5 & 7550 & $34.8^{\circ}$ & $1.3^{\circ}$ \\
\hline $10 \mathrm{~m}$ (std) & 29.8 & $2.23 \mathrm{~m}$ & $0.07 \mathrm{~m}$ & 6.5 & 1598 & $22.8^{\circ}$ & $3.2^{\circ}$ \\
\hline
\end{tabular}

Table 2. Path planning results using all the scans collected during the 2007 testing season. Destinations selected randomly at $5 \mathrm{~m}$ and $10 \mathrm{~m}$ from the origin of the scan.

Figures 6 and 7 present a sequence of snapshots from an Over-the-Horizon navigation experiment. Common in all figures is the global path which guides the robot around the steeper parts of the hill, avoiding large obstacles and drop-offs. The first scan (Fig. 6a) is used to segment the global path and find the first way-point. Then, a local path is planned using the local scan. The process is repeated in figures $6 \mathrm{~b}, \mathrm{c}$ and $6 \mathrm{a}, \mathrm{b}^{5}$. Finally, figure $7 \mathrm{c}$ contains the global path, the recorded 3D trajectory as estimated from the IMU and the wheel-odometry, and the pure wheel odometry, which deviates early on, and goes outside the terrain. Even though there was no accurate ground truth measurements, a qualitative error of approximately $1 \mathrm{~m}$ was observed. More specifically, the perimeter of the testing grounds is marked every $3 \mathrm{~m}$, thus every time the robot reached its final destination a tape measure was taken manually recording the reported errors.

\section{Conclusions}

In this paper we presented an overview of experimental results on autonomous Over-the-Horizon navigation performed in a Mars-like terrain. Over the last two years a large number of data was collected in realistic conditions which enabled us to fine-tune our approach to planetary exploration. The sensor choice was confirmed based on the quality of the terrain reconstruction as well as the path-planning performance.

Two different LIDAR sensors were used with different range, fov, and accuracy characteristics. Both sensors though provided detailed scans of the environment allowing the capture of topographic details down to centimeter accuracy. The employment of ITMs for terrain modelling maintained the high levels of accuracy while at the same time reducing the data volume by $90 \%-95 \%$. Moreover, path planing performed as an $\mathrm{A}^{*}$ search on the dual graph of an ITM was efficient and took into consideration the different cost parameters such as distance travelled, roughness of the terrain, and slope of the traversed terrain. Multiple tests were performed using the terrain data collected verifying the quality of CSA's path planning approach.

The ability of planetary rovers to travel beyond their sensing horizon, autonomously, in a single command cycle will be critical in future Mars missions. Over the last two years at CSA several experiments were performed verifying the validity of our approach. Currently, an expansion of the Mars emulation terrain by a factor of four, together with the investigation of bigger mobility platforms would lead to further development of the planetary exploration capabilities that would enable future mission to Mars and the Moon.

\section{References}

1. Kitware inc. visualization toolkits. http://www.vtk.org, Website (accessed: September 2005)., 2005.

\footnotetext{
${ }^{5}$ Only the scans $1,2,3,6$, and 8 are displayed due to lack of space.
} 


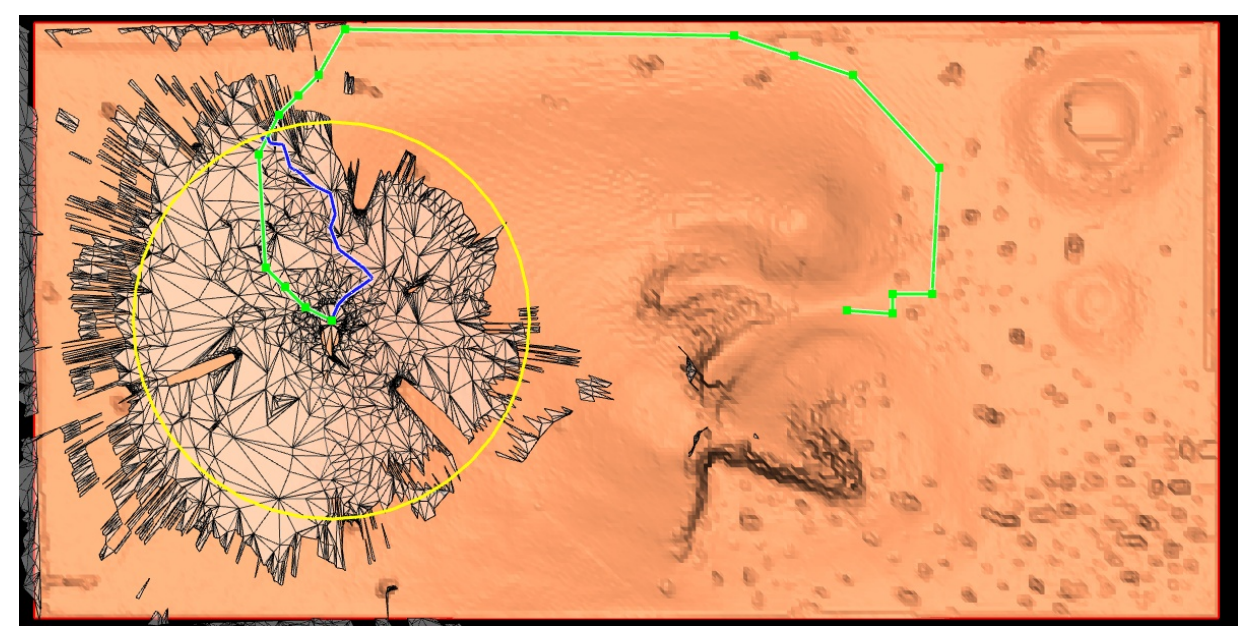

(a)

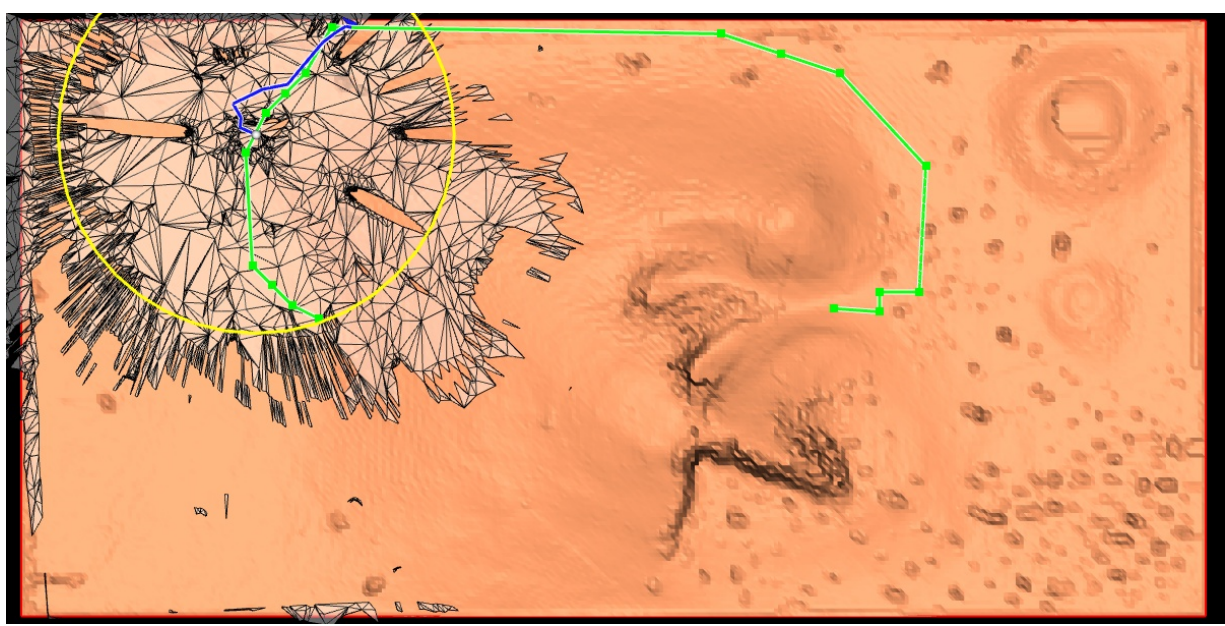

(b)

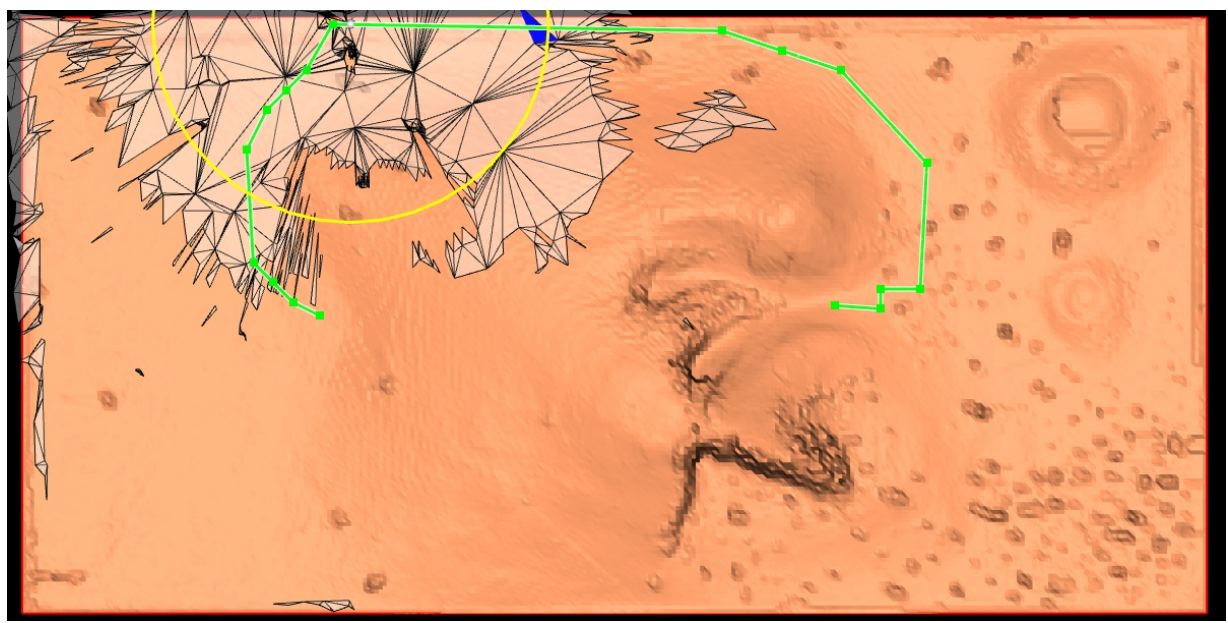

(c)

Fig. 6. (a)-(c) Selected steps through an autonomous Over-the-Horizon navigation experiment. At each step the global path (in green), the local scan, and the local path (in blue) are shown; the range used to segment the global path is indicated by a yellow circle. 


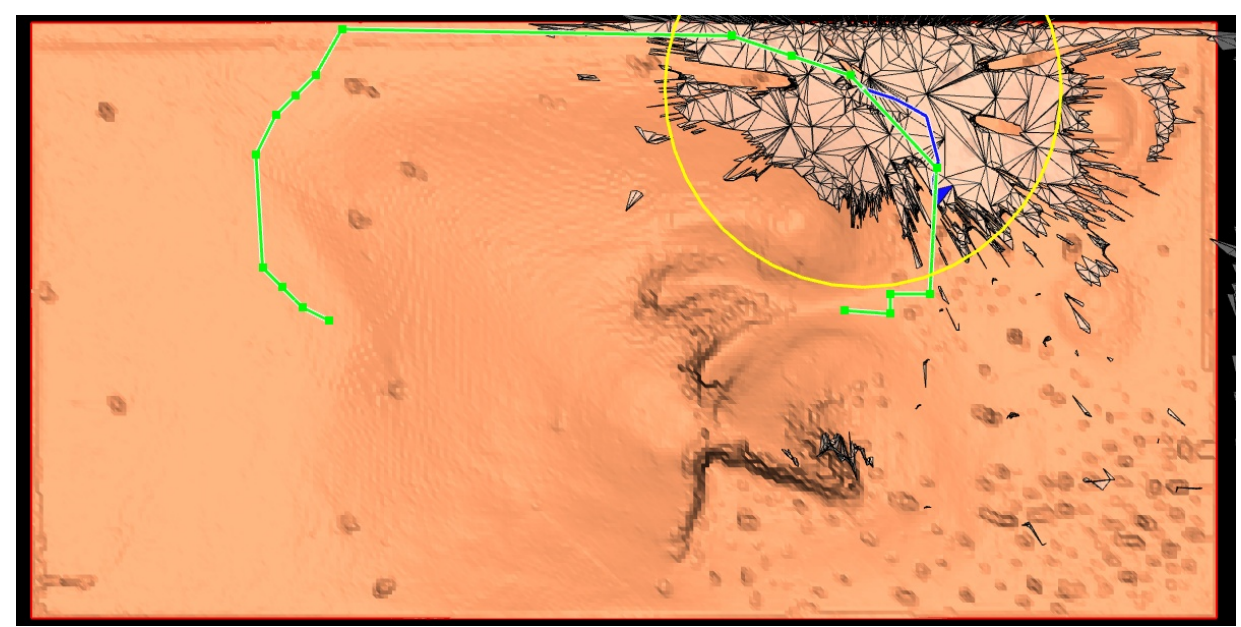

(a)

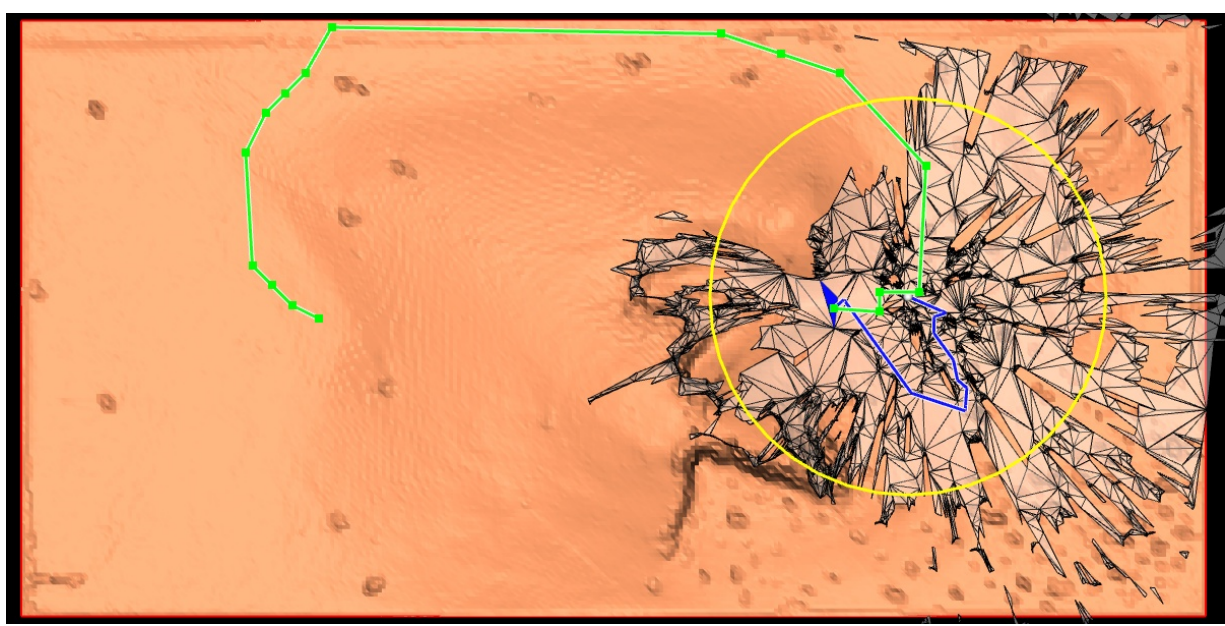

(b)

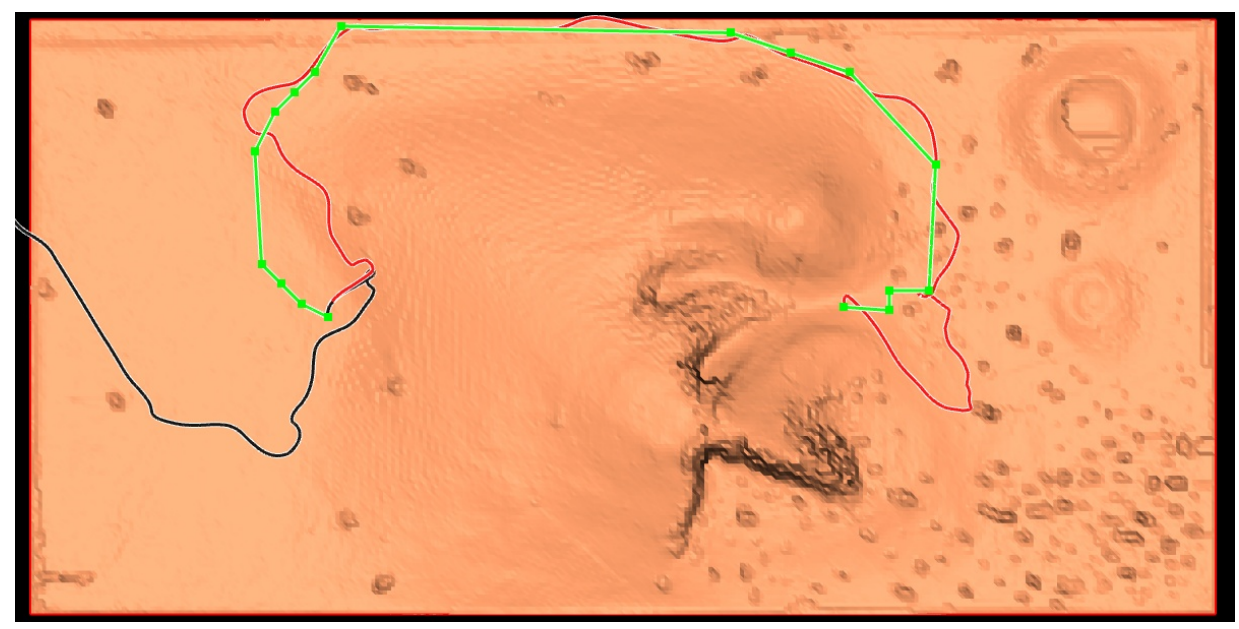

(c)

Fig. 7. (a),(b) Selected steps through an autonomous Over-the-Horizon navigation experiment. At each step the global path (in green), the local scan, and the local path (in blue) is shown; the range used to segment the global path is indicated by a yellow circle. (c) The Mars emulation terrain model, the global path (green), the recorded trajectory (red), and the pure odometry trajectory (shorter black line that deviates out of the figure). 
2. R. Alena, B. Gilbaugh, B. Glass, and S.P.Braham. Communication system architecture for planetary exploration. IEEE Aerospace and Electronic Systems Magazine, 16(11):4 - 11, Nov. 2001.

3. J. Bares, M. Hebert, T. Kanade, E. Krotkov, T. Mitchell, R. Simmons, and W.L. Whittaker. Ambler: An autonomous rover for planetary exploration. IEEE Computer, 22(6):18-26, June 1989.

4. J. Biesiadecki, C. Leger, and M. Maimone. Tradeoffs between directed and autonomous on the mars exploration rovers. In Procs. of Int. Symposium of Robotics Research, San Francisco, 2005.

5. B. Delaunay. Sur la sphère vide. Izvestia Akademii Nauk SSSR, Otdelenie Matematicheskikh i Estestvennykh Nauk, 7:793-800, 1934.

6. A. Kelly et al. Toward reliable off-road autonomous vehicles operating in challenging environments. The Int. Journal of Robotics Research, 25(5-6):449-483, June 2006.

7. David Wettergreen et al. Second experiments in the robotic investigation of life in the atacama desert of chile. In 8th Int. Symposium on Artificial Intelligence, Robotics and Automation in Space, Sept. 2005.

8. R. J. Fowler and J. J. Little. Automatic extraction of irregular network digital terrain models. In SIGGRAPH '79: Procs. of the 6th annual conference on Computer graphics $\&$ interactive techniques, pp. 199-207, 1979.

9. Daniel M. Gaines, Tara Estlin, and Caroline Chouinard. Spatial coverage planning and optimization for a planetary exploration rover. In 5th Int. Workshop on Planning and Scheduling for Space, 2003.

10. G. Giralt and L. Boissier. The french planetary rover vap: Concept and current developments. In Procs. of the 1992 lEEE/RSJ Int. Conf. on Intelligent Robots and Systems, v. 2, pp. 1391-1398, 1992.

11. S.B. Goldberg, M.W. Maimone, and L. Matthies. Stereo vision and rover navigation software for planetary exploration. In IEEE Aerospace conference proceedings, v. 5, pp. 2025-2036, Big Sky, MT, USA, March 2002.

12. M. Hebert, C. Caillas, E. Krotkov, I. Kweon, and T. Kanade. Terrain mapping for a roving planetary explorer. In Proc. of the IEEE Int. Conf. on Robotics and Automation, v. 2, pp. 997-1002, May 1989.

13. A. M. Howard and E. W. Tunstel, editors. Intelligence for Space Robotics, chapter MER Surface Navigation and Mobility. TSI Press, 2006.

14. T. Kubota, R. Ejiri, Y. Kunii, and I. Nakatani. Autonomous behavior planning scheme for exploration rover. In Second IEEE Int. Conf. on Space Mission Challenges for Information Technology, page 7, 17-20 July 2006.

15. Y. Kunii, S. Tsuji, and M. Watari. Accuracy improvement of shadow range finder: Srf for 3d surface measurement. In Procs. off IEEE/RSJ Int. Conf. on Intelligent Robots and Systems, v. 3, pp. 3041 - 3046, 27-31 Oct. 2003.

16. S.L. Laubach and J.W. Burdick. An autonomous sensor-based path-planner for planetary microrovers. In IEEE Int. Conf. on Robotics \& Automation, v. 1, pp. 347 - 354, May 1999.

17. C. Lee, R.L. Alena, T. Stone, J. Ossenfort, E. Walker, and H. Notario. Software architecture of sensor data distribution in planetary exploration. In IEEE Aerospace Conf., page 9, 4-11 March 2006.

18. M. Maimone, J. Biesiadecki, E. Tunstel, Y. Cheng, and C. Leger. Intelligence for Space Robotics, chapter Surface Navigation and Mobility Intelligence on the Mars Exploration Rovers, pp. 45-69. TSI press, 2006.

19. L. Matthies and Steven Shafer. Error modeling in stereo navigation. IEEE Journal of Robotics and Automation, $3(3): 239-250$, June 1987.

20. M. Montemerlo, S. Thrun, H. Dahlkamp, D. Stavens, and S. Strohband. Winning the darpa grand challenge with an ai robot. In Proc. of the AAAI National Conf. on Artificial Intelligence, Boston, MA, 2006.

21. A.I. Mourikis, N. Trawny, S.I. Roumeliotis, A. Johnson, and L. Matthies. Vision-aided inertial navigation for precise planetary landing: Analysis and experiments. In Proc. Robotics: Science and Systems, Jun. 2007.

22. Ioannis Rekleitis, Jean-Luc Bedwani, and Erick Dupuis. Over-the-horizon, autonomous navigation for planetary exploration. In Proc. of IEEE/RSJ Int. Conf. on Intelligent Robots and Systems, pp. 2248-2255, San Diego, California, USA, Oct. 2007.

23. Ioannis Rekleitis, Jean-Luc Bedwani, Erick Dupuis, and Pierre Allard. Path planning for planetary exploration. In 5th Canadian Conf. on Computer and Robot Vision, pp. 61-68, Windsor, ON., 28-30 May 2008.

24. M.G. Slack. Navigation templates: mediating qualitative guidance and quantitative control in mobile robots. IEEE Trans. on Systems, Man and Cybernetics, 23(2):452 - 466, March-April 1993.

25. N. Trawny, A.I. Mourikis, S.I. Roumeliotis, A.E. Johnson, and J.F. Montgomery. Vision-aided inertial navigation for pin-point landing using observations of mapped landmarks. Journal of Field Robotics, 24(5):257-378, 2007.

26. J. Vago. Overview of exomars mission preparation. In 8th ESA Workshop on Advanced Space Technologies for Robotics \& Automation, Noordwijk, The Netherlands, November 2004.

27. R. Volpe. Rover functional autonomy development for the mars mobile science laboratory. In IEEE Aerospace Conf., Big Sky, MT, USA, 2006.

28. J. Wright, A. Trebi-Ollennu, F. Hartman, B. Cooper, S. Maxwell, J. Yen, and J. Morrison. Terrain modelling for in-situ activity planning and rehearsal for the mars exploration rovers. In IEEE Int. Conf. on Systems, Man and Cybernetics, v. 2, pp. 1372 - 1377, 2005.

29. L. Yenilmez and H. Temeltas. Autonomous navigation for planetary exploration by a mobile robot. In Procs. of Int. Conf. on Recent Advances in Space Technologies, pp. 397-402, 20-22 Nov. 2003. 